\title{
Article
}

\section{Electrostatic Screening and Charge Correlation Effects in Micellization of lonic Surfactants}

Arben Jusufi, Antti-Pekka Hynninen, Mikko Haataja, and Athanassios Z. Panagiotopoulos J. Phys. Chem. B, 2009, 113 (18), 6314-6320• DOI: 10.1021/jp901032g • Publication Date (Web): 10 April 2009

Downloaded from http://pubs.acs.org on May 5, 2009

\section{More About This Article}

Additional resources and features associated with this article are available within the HTML version:

- $\quad$ Supporting Information

- $\quad$ Access to high resolution figures

- $\quad$ Links to articles and content related to this article

- Copyright permission to reproduce figures and/or text from this article

\section{View the Full Text HTML}




\title{
Electrostatic Screening and Charge Correlation Effects in Micellization of Ionic Surfactants
}

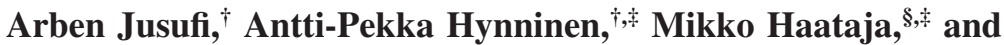 \\ Athanassios Z. Panagiotopoulos*, \\ Department of Chemical Engineering, Department of Mechanical and Aerospace Engineering, and Institute for \\ the Science and Technology of Materials, Princeton University, Princeton New Jersey 08544
}

Received: February 4, 2009; Revised Manuscript Received: March 12, 2009

\begin{abstract}
We have used atomistic simulations to study the role of electrostatic screening and charge correlation effects in self-assembly processes of ionic surfactants into micelles. Specifically, we employed grand canonical Monte Carlo simulations to investigate the critical micelle concentration $(\mathrm{cmc})$, aggregation number, and micellar shape in the presence of explicit sodium chloride $(\mathrm{NaCl})$. The two systems investigated are cationic dodecyltrimethylammonium chloride (DTAC) and anionic sodium dodecyl sulfate (SDS) surfactants. Our explicit-salt results, obtained from a previously developed potential model with no further adjustment of its parameters, are in good agreement with experimental data for structural and thermodynamic micellar properties. We illustrate the importance of ion correlation effects by comparing these results with a Yukawa-type surfactant model that incorporates electrostatic screening implicitly. While the effect of salt on the cmc is well-reproduced even with the implicit Yukawa model, the aggregate size predictions deviate significantly from experimental observations at low salt concentrations. We attribute this discrepancy to the neglect of ion correlations in the implicit-salt model. At higher salt concentrations, we find reasonable agreement of the Yukawa model with experimental data. The crossover from low to high salt concentrations is reached when the electrostatic screening length becomes comparable to the headgroup size.
\end{abstract}

\section{Introduction}

Self-assembly of amphiphilic molecules is based on the interplay of solvophilic and solvophobic interactions. Prominent examples are surfactants and lipid molecules, where the headgroup is typically hydrophilic and the tail consists of hydrophobic segments. Above a certain concentration, the amphiphiles aggregate into micelles or bilayer structures, ${ }^{1,2}$ when the energetic gain exceeds the entropic cost of aggregation. The self-assembly process depends on the amphiphile and solvent molecular architectures, but also on temperature and the presence of other solution components such as dissolved salts. ${ }^{1-6} \mathrm{Am}-$ phihiles are used in various applications, from cleaning products ${ }^{3,4}$ to nanotechnology. ${ }^{7,8}$ In nature, lipids are the most important components of biological cell membranes. ${ }^{1,6,9}$

The general mechanism of self-assembly is well-understood. However, the role of specific interactions on the molecular scale and their influence on the critical association concentration and the structure of aggregates in solution are still major points of interest. A convenient approach to investigate such problems is through molecular simulations. ${ }^{10}$ Atomistic molecular dynamics (MD) simulations have been used to study surfactant micelles ${ }^{11-24}$ and lipid membranes. ${ }^{25-31}$ With fully atomistic simulations the self-assembly process itself is difficult to model, due to the large computational effort required to reach time scales of microseconds relevant for micellization. ${ }^{32}$ Implicitsolvent models are helpful in reducing the computational cost of simulations. ${ }^{33-42}$ In comparison with atomistic simulations, one can save 2 orders of magnitude in computation time using implicit-solvent models, ${ }^{42}$ thus enabling self-assembly studies

\footnotetext{
* To whom correspondence should be addressed. E-mail: azp@ princeton.edu.

${ }^{\dagger}$ Department of Chemical Engineering.

* Institute for the Science and Technology of Materials.

${ }^{\S}$ Department of Mechanical and Aerospace Engineering.
}

on longer time and length scales. We recently developed such an implicit-solvent model that is capable of reproducing the experimental critical micelle concentration $(\mathrm{cmc})$ and the aggregate structures of ionic surfactants, such as sodium dodecyl sulfate (SDS) and dodecyltrimethylammonium chloride (DTAC). The approach was found to be transferable to related ionic surfactants of different headgroups and tail lengths. ${ }^{42}$

In many implicit-solvent models of amphiphilic molecules, electrostatic interactions between charged species have been treated with a Debye-Hückel or Yukawa potential that accounts for Coulomb screening. ${ }^{43-49}$ This approach reduces the computational cost, allowing large-scale investigations of structural properties. Similar methods have also been used in protein simulations. ${ }^{50-52}$ Yukawa models in ionic systems are justified at low Coulomb couplings where the underlying linear Poisson-Boltzmann (PB) theory is valid and charge correlation effects are not important. The question arises, however, whether simple Yukawa models of salt-mediated interactions between amphiphiles can describe aggregate formation, thermodynamics, and micellar structure in simulations. In other words, how important are charge correlation effects for the self-assembly process? In this work we address this question by using the micellization of ionic surfactants as representative of selfassembly processes of building blocks with ionic interactions. We do this by comparing a Yukawa-type model to an explicitsalt model with respect to their ability to predict the $\mathrm{cmc}$ and the aggregation number of the micelles as a function of added salt. In both cases, we use as starting point a surfactant model developed for the salt-free case in ref 42 and study added-salt cases with no adjustment to any of the model parameters. We show that electrostatic correlation effects become important at low ionic strengths, where the Yukawa model fails to describe realistically the micellization process. Furthermore, we demonstrate for the first time that our explicit-ion model is capable 
of predicting an accurate salt dependence of the $\mathrm{cmc}$, aggregate size, and micellar shapes. At very high salt and surfactant concentration we observe the occurrence of cylindrical micelles, again in agreement with experiment.

The structure of this paper is as follows. In the next section we give a brief description of the methods that were used in the simulations and the main features of the implicit-solvent model. The results are summarized in section 3 , where we discuss the micellization properties of SDS and DTAC in the presence of explicit $\mathrm{NaCl}$. The results are compared to the Yukawa-type potential with implicit salt. Finally, in section 4, we conclude and give a short outlook for further applications of the proposed explicit-ion model.

\section{Simulation Model and Methods}

Thermodynamic properties were determined using grand canonical Monte Carlo simulations, in which both the number of surfactants and the number of salt ions were allowed to fluctuate, while the volume and the temperature were fixed. The cmc was obtained as the point of maximum change in slope of the pressure-surfactant concentration equation of state. ${ }^{39,42}$ Unless explicitly specified, simulation runs were performed in cubic boxes of length $L=6.1 \mathrm{~nm}$. We used histogram reweighting techniques ${ }^{53-55}$ to circumvent the strong hysteresis effects $^{56}$ at the target temperature of 298 K. Specifically, we performed a series of runs at a series of chemical potentials and temperatures up to $402 \mathrm{~K}$. At each run the number of surfactants, the number of co-ions, and the total energy were recorded in a histogram. Using the histogram reweighting technique, we combined the histograms and determined the equation of state at the target temperature of $298 \mathrm{~K}$.

We used a combination of MC moves: $25 \%$ of the moves were deletions/insertions of surfactants and salt ions, $20 \%$ were displacements/rotations of surfactants, $10 \%$ were used to regrow the surfactants, $15 \%$ were ion displacements, and $5 \%$ were cluster moves. In the insertion/deletion moves charge neutrality was ensured by always pairing the insertion/deletion of a surfactant or co-ion with that of a counterion. Regrowth and chain insertion/deletion were carried out using configurational bias techniques. ${ }^{57}$ Electrostatics were treated with the Ewald method $^{58}$ using 518 Fourier-space vectors and a real-space cutoff of $3.05 \mathrm{~nm}$. Typical runs comprised 10-30 million MC moves for equilibration, and, depending on the system, 50-100 million $\mathrm{MC}$ moves for production. The longer runs were carried out for systems with larger aggregate sizes.

We used the implicit-solvent model developed in our previous work $^{42}$ for the salt-free case. In ref 42 we parametrized the hydrophobic potential between the tail beads by matching the $\mathrm{cmc}$ and the aggregate size of SDS to experimental values. The simultaneous matching of the $\mathrm{cmc}$ and the aggregate size was made possible by concentrating the hydrophobic interaction between the terminal (i.e., furthest from the headgroup) tail beads only. This also makes it favorable for the surfactants to aggregate into concentric objects; i.e., the chains are buried inside the micelles, whereas the headgroups are mainly placed on the surface. We note that the model reproduces the liquidlike characteristics of the micelle interior, in agreement with atomistic simulation ${ }^{19,22}$ and experimental results. ${ }^{59}$

The interactions between all other tail beads (i.e., except terminal beads) were taken from the OPLS force field. ${ }^{60} \mathrm{We}$ used the atomistic settings for the bond potentials ${ }^{13,22}$ and did not coarse-grain the tail beads. The headgroup and counterion interactions were described by effective potentials that account for various force contributions: electrostatics, hydration, disper- sion, and short-range repulsion. In the headgroup-counterion interaction a Coulomb correction was employed to account for close distance dependence of the dielectric permittivity. The parameter values for these interactions are given in ref 42 . We note, however, that the prefactor of the electrostatic potential contributions, the Bjerrum length $\lambda_{\mathrm{B}}=e^{2} /(4 \pi \epsilon k T)$ (dielectric constant $\epsilon$, elementary charge $e$, and thermal energy $k T$ ), was set to $0.71 \mathrm{~nm}$ instead of the experimental value of $0.72 \mathrm{~nm}$ at the temperature of $298 \mathrm{~K}$. In the present study, we used the experimental value and recalculated the quantities shown in ref 42, particularly SDS and DTAC in the salt-free case. Apart from this minor correction, no other modification of the model parameters shown in ref 42 was made. For the interaction between the $\mathrm{NaCl}$ salt ions we used the parametrization given in ref 61 . In ref 42 we also demonstrated the transferability of the SDS surfactant model to cationic surfactants (DTAB, DTAC) and sodium alkyl sulfate with alkyl chain lengths between 6 and 14.

In our Yukawa model, the surfactant tails interact as just described, with potential parameters obtained from ref 42 . The headgroups interact with each other via a short-range repulsive Weeks-Chandler-Anderson (WCA) potential ${ }^{62}$ and the screened Coulomb potential. The WCA interaction accounts for the finite size of the headgroup. The energy scale is $2.5 \mathrm{~kJ} / \mathrm{mol}$ at the target temperature of $298 \mathrm{~K}$ and is roughly equal to the thermal energy. Headgroup-tail WCA interactions are obtained using the Lorentz-Berthelot combining rule. The Coulomb screening is modeled through a Yukawa/Debye-Hückel potential

$$
V_{\text {yuk }}(r)=\frac{e^{2}}{4 \pi \epsilon} \frac{\exp \left(-\kappa\left(r-\sigma_{\mathrm{WCA}}\right)\right)}{r},
$$

where $\kappa=\left[2 e^{2} c /(\epsilon k T)\right]^{1 / 2}$ is the inverse screening length and $c$ is the total counterion concentration. The counterion concentration $c$ is given by the sum of the salt and surfactant concentrations as

$$
c=\mathrm{cmc}_{0}+c_{\mathrm{s}}
$$

The only unspecified parameter is the headgroup diameter $\sigma_{\mathrm{WCA}}$. The value was obtained by fitting the $\mathrm{cmc}$ to the salt-free case. In eq 2 we used $\mathrm{cmc}_{0}=8 \mathrm{mM}$ for SDS and $\mathrm{cmc}_{0}=20 \mathrm{mM}$ for DTAC $\left(c_{\mathrm{s}}=0\right)$. These values are in the experimental range, namely, $7.7^{63}$ to $9.3 \mathrm{mM}^{64}$ for SDS and $17.2^{65}$ to $21.3 \mathrm{mM}^{66}$ for DTAC. Best fits for the cmc were obtained with $\sigma_{\mathrm{WCA}}=$ $0.44 \mathrm{~nm}$ for SDS which corresponds to the smallest headgroupheadgroup distance that we measured in separate atomistic MD simulations of headgroups and counterions. ${ }^{42}$ For DTAC we used $\sigma_{\mathrm{WCA}}=0.65 \mathrm{~nm}$, a value that is somewhat larger than the minimum separation between the headgroups (around $0.55 \mathrm{~nm}$ ). These diameter values were used in the added-salt cases as well.

\section{Results}

Using explicit-ion simulations in the salt-free solution, we obtained $\mathrm{cmc}_{0}=8.4 \pm 0.3 \mathrm{mM}$ for SDS and $\mathrm{cmc}_{0}=16.7 \pm$ $0.5 \mathrm{mM}$ for DTAC. The results are close to the ones reported earlier. ${ }^{42}$ The difference is due to a larger box length used in the present study, $L=6.1 \mathrm{~nm}$ (compared to $L=4.6 \mathrm{~nm}$ ), and the correction of the Bjerrum length, $\lambda_{\mathrm{B}}$, to the experimental value of $0.72 \mathrm{~nm}$ instead of $0.71 \mathrm{~nm}$ used in the previous study. A larger box was used here since we expect bigger aggregates at high salt concentrations. While the cmc's are slightly affected (12-15\% smaller than in ref 42) by the correction of $\lambda_{B}$, the aggregation numbers remain unchanged. Our simulated cmc's agree with experimental results that lie between $7.7^{63}$ and 9.3 $\mathrm{mM}^{64}$ for SDS and between $17.2^{65}$ and $21.3 \mathrm{mM}^{66}$ for DTAC. 


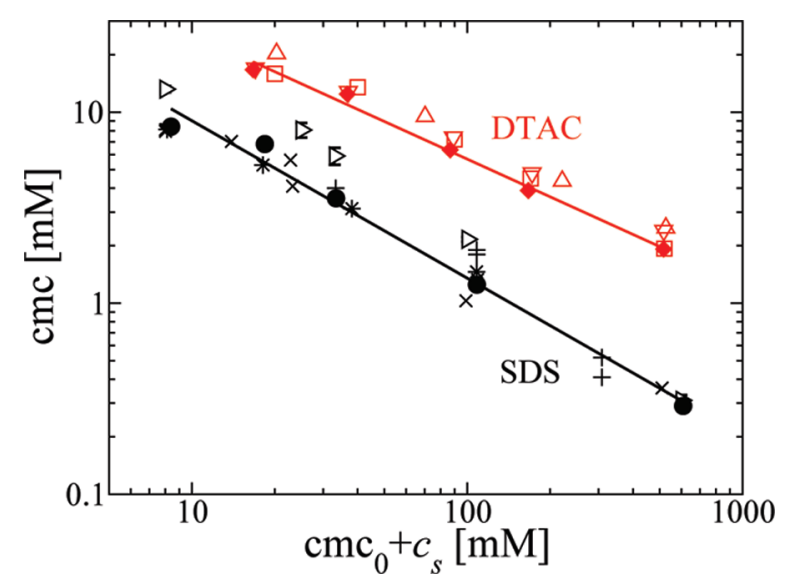

Figure 1. $\mathrm{cmc}$ as a function of the total counterion concentration $\mathrm{cmc}_{0}$ $+c_{\mathrm{s}}$. For SDS (black line and symbols): explicit-ion simulations (solid circles), Yukawa model (open right-pointing triangles), and experimental data from ref 2 (asterisks), ref 67 (plus signs), and ref 68 (times signs). For DTAC (red line and symbols): explicit-ion simulations (solid diamonds), Yukawa model (open boxes), and experimental data from ref 69 (open downward-pointing triangles) and ref 70 (open upwardpointing triangles). The lines are linear least-squares fits to the explicition results. Error bars are comparable to the symbol sizes.

We note that the DTAC results are pure predictions. The DTAC tail parameters are gained from the SDS parametrization, including the hydrophobic energy parameter, while the parametrization of the headgroup ion interaction potentials is based on fitting of the pair correlation functions obtained from separate atomistic simulations; see ref 42 for further details. The pair correlation functions of the ion systems are not affected by the small change in the Bjerrum length.

For the Yukawa-SDS model we obtain $\mathrm{cmc}_{0}=13.2 \pm 0.5$ $\mathrm{mM}$, which is somewhat above the experimental range. A better result would have been obtained if we had used a headgroup diameter smaller than $\sigma_{\mathrm{WCA}}=0.44 \mathrm{~nm}$, which would weaken the effective headgroup repulsion further. Smaller values of $\sigma_{\mathrm{WCA}}$, however, would have been unrealistic. For the YukawaDTAC model we obtain $\mathrm{cmc}_{0}=15.9 \pm 1.0 \mathrm{mM}$, which is closer to the lower experimental range.

Addition of salt leads to a significant drop in the $\mathrm{cmc}$ in line with the empirical power-law salt dependence observed for many ionic surfactants, ${ }^{2}$ as shown in Figure 1. The explicit-ion simulation results agree very well with experimental data over the whole salt concentration range, i.e., over 2 orders of magnitude. The model is robust with respect to headgroup change from SDS to DTAC without any adjustment of the tail bead parameters. As already stated, model parameters were taken unmodified from ref 42; the excellent agreement between simulations and experiments has not been forced. In the same figure we also plot results from the Yukawa model. The overall agreement with experiments is again reasonable and demonstrates that even the simple Yukawa model is able to reproduce the dependence of the $\mathrm{cmc}$ on salt concentration, despite some deviations particularly for SDS.

We computed the aggregate size $M$ by defining the aggregate with a simple cutoff criterion: two surfactants belong to the same micelle if the center of mass of one tail is separated by less than $0.4 \mathrm{~nm}$ from that of another tail. This cutoff value was tested to give the right aggregation number by visual inspection of representative configurations. Figure 2 shows the salt dependence of the mean aggregation number $\langle M\rangle$. Without added salt, we obtain values similar to those reported in ref $42 .\langle M\rangle$ increases with increasing salt concentration $c_{\mathrm{s}}$, in agreement with

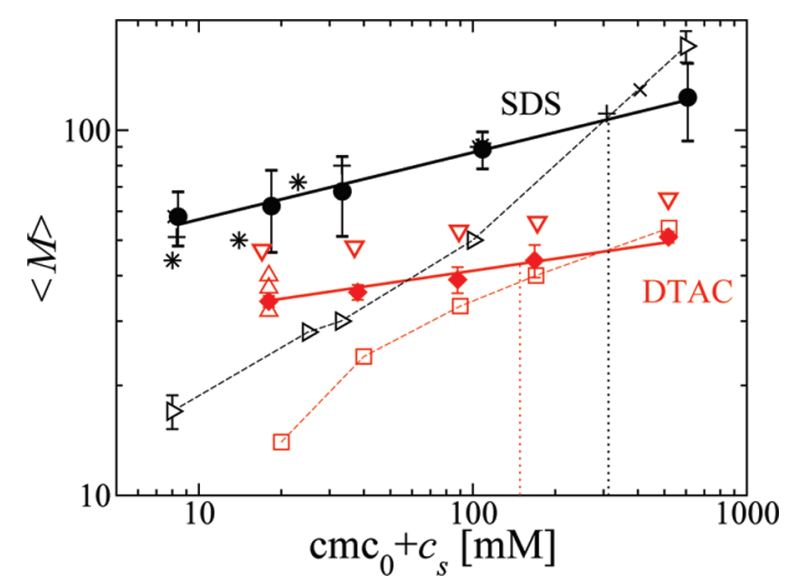

Figure 2. Mean aggregation number $\langle M\rangle$ as a function of total counterion concentration $\mathrm{cmc}_{0}+c_{\mathrm{s}}$. Symbols are the same as those for Figure 1. Solid lines are fits of the explicit-ion simulation results, and dashed lines are guides for the eye for the Yukawa model results. The dotted vertical lines mark the concentrations at which the screening length becomes smaller than the headgroup size for the corresponding surfactant.

experimental findings. SDS and DTAC explicit-ion results both exhibit a power law dependence, as seen experimentally for many ionic surfactants related to the ones studied here..$^{72-75} \mathrm{In}$ particular for SDS explicit-ion results, the agreement with experimental results is good. For DTAC, the simulation model systematically underestimates the experimentally observed aggregation number in the added salt cases. The reason for the underestimation could lie in the overestimation of the DTAC headgroup size. The headgroup parameters of the implicit model result from matching of the headgroup counterion pair correlation function obtained from implicit-solvent simulations with the same correlations obtained from atomistic simulations. There are many possible error sources: from the quality of atomistic ion force field parameters to the assumption that the implicitsolvent interaction parameters in bulk are used in the micellar system without further adjustments. Despite these uncertainties, the deviations for the aggregation numbers are always less than $20 \%$.

In contrast to the explicit-salt results, the Yukawa model does not reproduce the salt dependence of the aggregation number, as seen in Figure 2. At low salt concentrations, $\langle M\rangle$ predicted from the Yukawa model is much smaller than experimental values. Above a certain salt concentration the agreement with experiments and explicit-ion simulation results becomes better. The position of the threshold salt concentration is different for SDS and DTAC. We interpret this as being due to the different headgroup sizes of the surfactants. The effective (hydrated) diameter for the SDS headgroup is $\sigma=0.54 \mathrm{~nm}$ and for DTAC headgroup $\sigma=0.79 \mathrm{~nm}$ in our explicit-ion model. These values were obtained by matching pair correlation functions obtained from implicit-water simulations of headgroups and counterions with atomistic explicit-water simulation data. ${ }^{42}$ At low salt concentrations the screening length $\kappa^{-1}$ is much larger than $\sigma$. The screening length becomes comparable to the headgroup size; i.e., $\kappa \sigma \approx 1$ around $150 \mathrm{mM}$ for DTAC and $315 \mathrm{mM}$ for SDS, as indicated by vertical dotted lines in Figure 2. Interestingly, at these and higher salt concentrations the Yukawa model seems to reproduce experimental values for the aggregate size, as can be particularly seen for DTAC. The Yukawa results of SDS exhibit scatter at high salt concentration $(600 \mathrm{mM})$ in Figure 2, but the trend is clearly observable.

The hypothesis put forward based on these results is that the $\mathrm{cmc}$ is mainly determined by the strength of the hydrophobic 


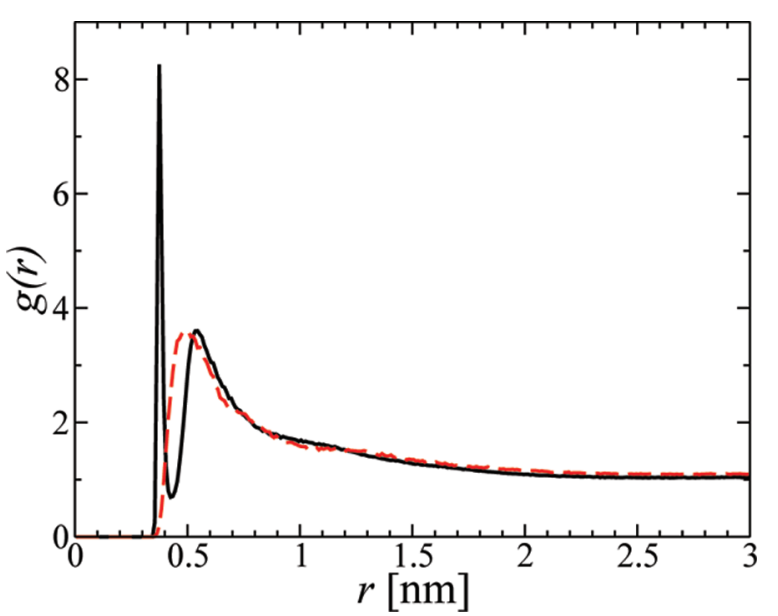

Figure 3. Pair correlation function $g(r)$ between headgroups and counterions for SDS (black solid line) and DTAC (red dashed line) as a function of their separation $r$ without added salt.

interaction and of the effective repulsion between the headgroups, which contains Coulomb screening. The $\mathrm{cmc}$ is the concentration at which micelles start forming. Up to the $\mathrm{cmc}$, the system is homogeneous and the screening parameter $\kappa$ reasonably accounts for Coulomb screening effects. However, when micelles are formed, i.e., above the $\mathrm{cmc}$, local headgroup counterion correlations enhance electrostatic screening. These effects are not captured by the simple Yukawa and similar meanfield models. This is particularly important for salt concentrations at which $\kappa \sigma \lesssim 1$. Above this threshold the screening length $\kappa$ is smaller than the effective headgroup size and larger micelles can form. The Yukawa and explicit-salt surfactants behave similarly in this regime. Micellar growth in the Yukawa surfactant system at low salt is limited by the large effective headgroup size.

To have a measure for the headgroup counterion correlations, we computed the degree of counterion binding to headgroups at different salt concentrations. Figure 3 shows the counterionheadgroup pair correlation function $g(r)$ for SDS and DTAC in the salt-free case, where only headgroup and counterions are present. The behavior of $g(r)$ for the added salt cases remains qualitatively similar, with only the peak heights changing with respect to the salt-free case. As discussed in detail in ref 42 , the headgroup-counterion correlation for SDS possesses a double peak profile, accounting for first and second counterion coordination shells of headgroups due to hydration layers around the ions.

Those effects have been observed in atomistic simulations as well, although they differ quantitatively, which in turn lead to different degrees of counterion binding in the corresponding shells. ${ }^{19-22}$ The degree of counterion binding $\xi$ has been estimated from the fraction of counterion under the peaks up to the subsequent minimum. In the DTAC system the hydration layers of the ions are strong and the cutoff distance is large, around $1.05 \mathrm{~nm}$. For SDS the first and second minima are at 0.43 and $0.92 \mathrm{~nm}$, yielding a corresponding first and second degree of counterion binding $\xi_{1}$ and $\xi_{2}$, respectively. While the first peak position agrees well with atomistic results, ${ }^{18,20,22}$ the second peak position is somewhat bigger than observed in atomistic studies. This is due to a lack of a third coordination shell of water molecules around the ions in our implicit-water model. However, for the degree of counterion binding the area is of bigger importance than the exact peak position. In Table 1 , the degrees of counterion binding $\xi$ are given at different salt concentrations. According to atomistic studies, ${ }^{18,22}$ the vast
TABLE 1: Radius $R$ and Counterion Binding Values $\xi$ of SDS and DTAC Micelles for Various NaCl Concentration $c_{\mathrm{s}}{ }^{a}$

\begin{tabular}{lccccc}
\hline & $c_{\mathrm{s}}(\mathrm{mM})$ & $R(\mathrm{~nm})$ & $\xi_{1}$ & $\xi_{2}$ & $\xi$ \\
\hline \multirow{3}{*}{ SDS } & 0 & 2.02 & 0.11 & 0.56 & 0.67 \\
& 10 & 2.04 & 0.11 & 0.59 & 0.70 \\
& 25 & 2.09 & 0.13 & 0.60 & 0.73 \\
& 100 & 2.26 & 0.14 & 0.61 & 0.75 \\
DTAC & 600 & 2.48 & 0.19 & 0.73 & 0.92 \\
& 0 & 1.84 & & & 0.65 \\
& 20 & 1.83 & & & 0.69 \\
& 70 & 1.86 & & & 0.73 \\
& 150 & 1.93 & & & 0.78 \\
& 500 & 1.99 & & & 0.95
\end{tabular}

${ }^{a}$ For the SDS cases the counterion binding is distinguished between first and second shell binding, $\xi_{1}$ and $\xi_{2}$, respectively. DTAC micelles posses only second shell counterion binding.

majority of ion binding is through its second coordination shell of the headgroups. The total ion binding degree $\xi$ is around $67 \%$ in the absence of salt, which lies in the range of atomistic results which report values of $\xi$ between $50^{18}$ and $80 \% .^{22}$ Experimental values lie in a smaller interval, namely, between $62^{67}$ and $72 \% .{ }^{77}$ Addition of salt generally increases the ion binding, however, only weakly at modest salt concentration, i.e., below $100 \mathrm{mM}$. Within this range our values remain between 70 and $75 \%$. Experimental groups reported a similar trend. ${ }^{67,68}$ At high salt concentration experimental measurements obtained counterion binding of $86 \%$ already at $300 \mathrm{mM} .{ }^{67}$ One can expect a higher degree at $600 \mathrm{mM}$, where we obtained $92 \%$ binding; i.e., at this salt concentration the micelles are almost completely neutralized.

DTAC micelles possess similar characteristics. Only at 500 $\mathrm{mM}$ salt concentration the counterion binding reaches almost $100 \%$. At lower ionic strengths the degree increases from $65 \%$ in the salt-free case to almost $80 \%$ at $150 \mathrm{mM}$ salt concentration. The first value is in excellent agreement with experimental data $(63 \%) .{ }^{75}$ Calculated values that were used to match measured surface potentials report a slight salt dependence, ranging between $61 \%$ in the salt-free case to $82 \%$ at $c_{\mathrm{s}}=650 \mathrm{mM}$ $\mathrm{NaCl} .{ }^{76}$ Note that our model predicts only a second ion binding shell. This is a significant difference to SDS. A large headgroup size and a lack of a first ion binding shell reduce the probability of ion correlation effects, such as ion bridging observed in SDS micelles. ${ }^{18}$ In turn, the cmc of DTAC is larger and its micelle sizes smaller than SDS micelles, irrespective of addition of salt. A lack of such ion correlations also makes possible the use of Yukawa models, as long as $\kappa \sigma \gtrsim 1$. In the corresponding salt regimes we saw that the aggregate size of DTAC micelles is well-described by a Yukawa model, in contrast to the SDS micelles, where the Yukawa model predictions of the aggregate size is rather limited; see Figure 2.

In Table 1 we have given values for micellar radii obtained as the average distance between the headgroups and the center of mass of the micelles. For both cases, SDS and DTAC, the radius $R$ increases very weakly. Information on micellar shape is obtained from the principal moments of inertia $I_{1} \geq I_{2} \geq I_{3}$. In Figure 4 we plot the ratio $I_{1} / I_{3}$ as a function of the counterion concentration for SDS and DTAC. Representative snapshots are given in Figure 5.

Clearly, the asphericity of SDS micelles increases with increasing salt concentration. This is in line with experimental observations $\mathrm{s}^{2,78,79}$ and indicates a trend toward ellipsoidal shapes of the micelles. In many experimental studies this trend is pronounced. ${ }^{71,80,81}$ In contrast to SDS, the DTAC micelles remain spherical almost over the whole salt concentration range, again 


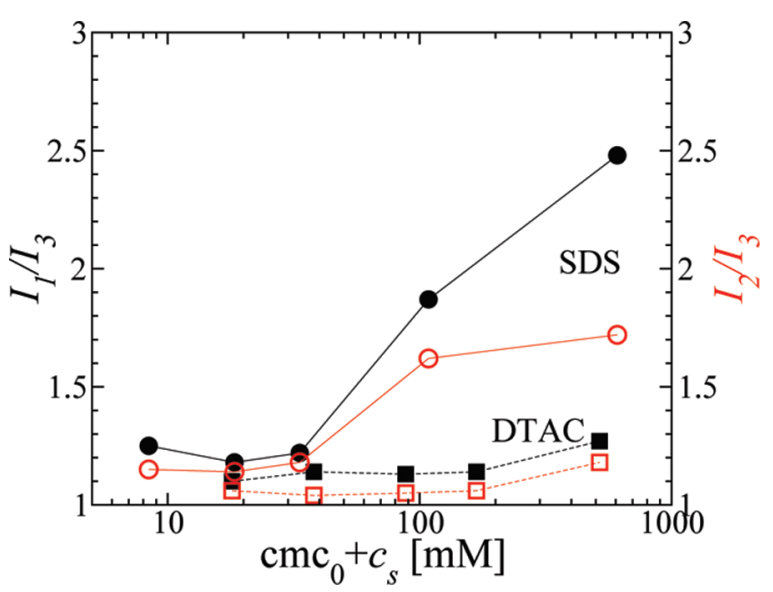

Figure 4. Ratio of moments of inertia $I_{1} / I_{3}$ and $I_{2} / I_{3}$ (where $I_{1} \geq I_{2} \geq$ $I_{3}$ ) for SDS (circles) and DTAC (squares) as function of ion concentration. While DTAC micelles remain almost spherical over the whole ion concentration range, SDS micelles become oblate ellipsoids at higher salt concentration. The lines serve as guides for the eye.

in line with experimental observations. ${ }^{82}$ This is quantified by the ratio $I_{2} / I_{3}$, which is similar to $I_{1} / I_{3}$, as can be seen from Figure 4. In contrast, SDS micelles possess an extension in two dimensions, indicating an oblate ellipsoidal shape. There are some results from scattering experiments contradicting this observation. ${ }^{81}$ However, as pointed out by Tanford, an oblate ellipsoid is the more favorable shape for this particular aggregate size. ${ }^{79}$ Cylindrical micelles are expected for aggregates with at least 150 surfactants; our micelles at the cmc are smaller. In fact, in recent work, ${ }^{78}$ it is stated that a definite conclusion regarding elongated ellipsoids (prolate ellipsoids) can only be made for solutions at high surfactant concentration and/or high ionic strength.

A sphere-to-rod transition, as experimentally observed in ref 81 , results from micellar fusion and requires simulations of multiple micelles at surfactant concentrations much above the cmc. For this purpose, we performed simulations of a SDS system at high surfactant and salt concentration. The number of SDS molecules was fixed at 370 in a cubic box with edge length of $10.7 \mathrm{~nm}$ which corresponds to a surfactant concentration of $500 \mathrm{mM}$. For the salt the chemical potential was fixed, corresponding to a $1 \mathrm{M}$ bulk $\mathrm{NaCl}$ concentration. At those conditions, formation of cylindrical micelles is experimentally observed. ${ }^{78,81}$ Beginning with a random configuration, the surfactants start forming smaller aggregates. Over time, those micelles fuse and form larger micelles, including a cylindrical one, as can be seen from the snapshot in Figure 6. We did not attempt to determine precisely the onset concentration for the sphere-to-rod transition because we are unable to reach equilibrium in this case; growth of the cylinder due to further micellar fusion is likely at much longer times. The snapshot shown in Figure 6 is taken after $4 \times 10^{8}$ MC moves. It suggests that the implicit-salt model favors formation of cylindrical micelles at high salt and surfactant concentrations. A question to be examined in future studies is the ability of the explicitsalt model to reproduce morphological transitions into more complex shapes, such as bilayers and inverse micelles for appropriate surfactant/solvent systems.

\section{Conclusions}

We investigated electrostatic screening effects on micellization of ionic surfactants by performing grand canonical Monte Carlo simulations of SDS and DTAC amphiphiles in the presence of explicitly and implicitly modeled $\mathrm{NaCl}$. We studied salt effects on the cmc and the aggregate structure, in particular the size and shape of micelles. For both surfactant types good agreement was achieved with experimental data of those quantities with the explicit-salt model, without adjustment of the model parameters developed in our earlier work. ${ }^{42}$ For the added-salt cases, the results obtained in this work are pure predictions that are in agreement with available experimental data.

In the simplified screened Coulomb model for the ionic headgroups of SDS and DTAC surfactants counterions are taken into account implicitly through the use of a Yukawa potential between the headgroups. The Yukawa model results show that reasonable agreement with experimental data is obtained for the salt dependence of the cmc. Agreement of the aggregation number results, however, was observed only at high salt concentration. The crossover occurs when the screening length $\kappa^{-1}$ becomes roughly comparable or smaller than the effective headgroup size $\sigma$.

This significant difference between explicit- and implicition results suggests that headgroup-counterion correlations
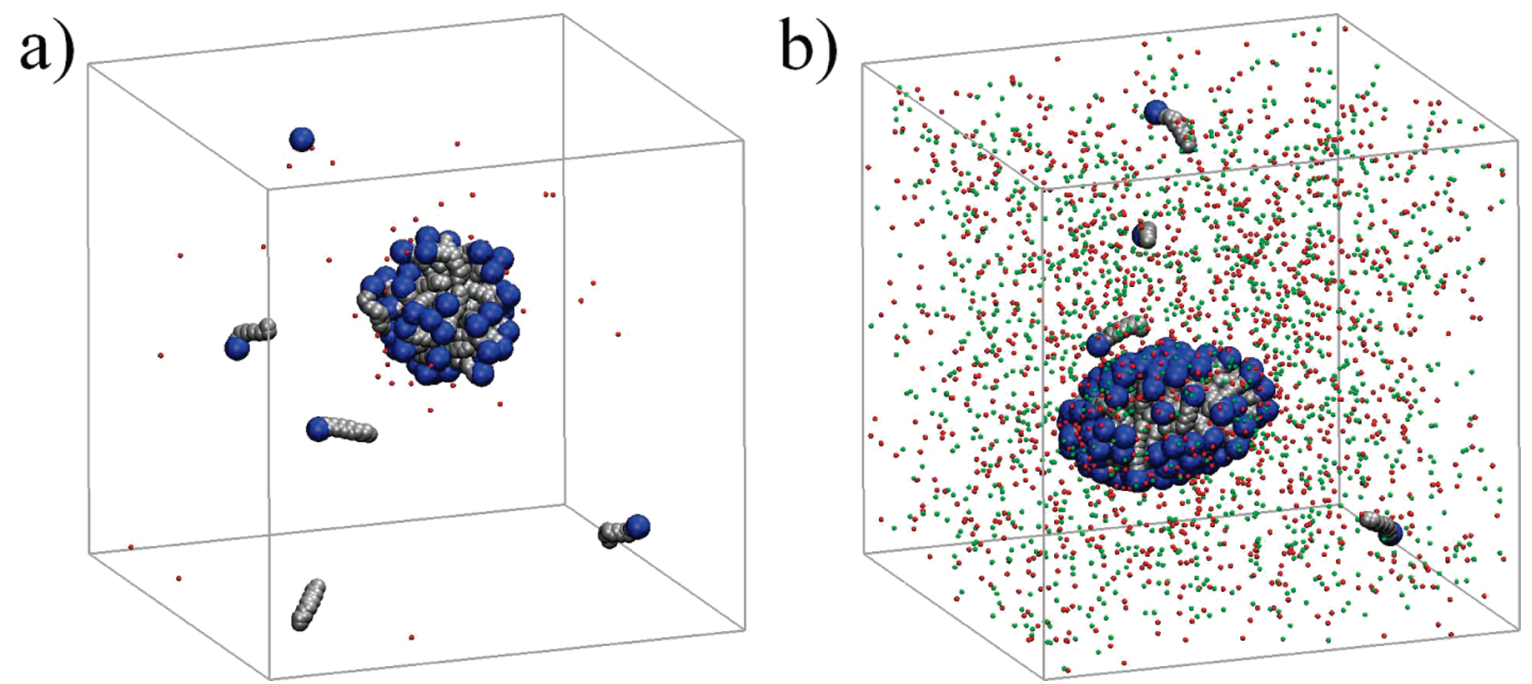

Figure 5. Snapshot of SDS micelles (a) in salt-free solution with $\langle M\rangle=51$, and (b) at $600 \mathrm{mM} \mathrm{NaCl}$ concentration with $\langle M\rangle=124$. Counterions and co-ions are represented by red and green dots, respectively. 


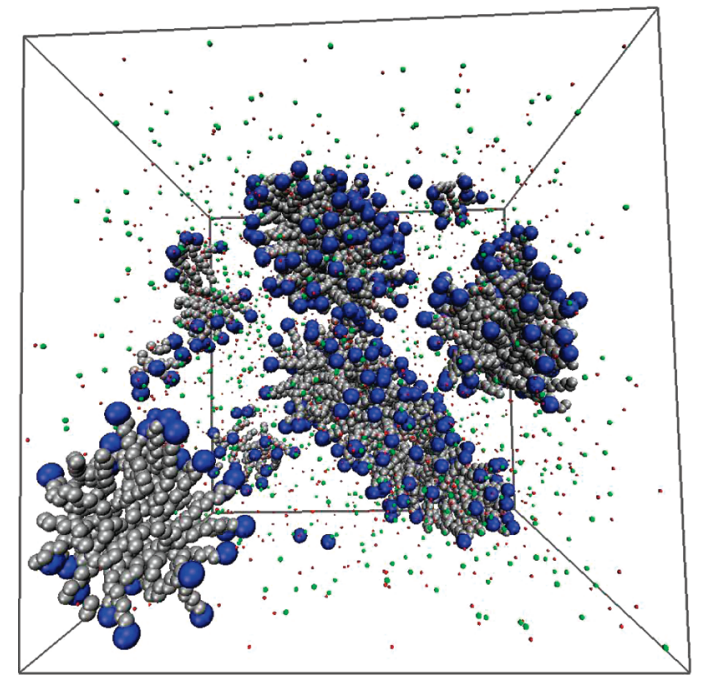

Figure 6. Snapshot of $0.5 \mathrm{M}$ SDS micelles in $1 \mathrm{M} \mathrm{NaCl}$. The starting configuration was random. One cylindrical micelle was formed through fusion of smaller aggregates and is located in the back-most half of the box. Counterions and co-ions are represented by red and green dots, respectively.

that are not captured by the screening parameter used in the Yukawa model are important in the self-assembly process at low salt. The effective screening is caused by the high degree of counterion binding that we observe even at low salt concentration. The discrepancy between the Yukawa and explicit-ion model increases below $\kappa \sigma \lesssim 1$. The use of Yukawa type models in the low salt regime becomes inconsistent in terms of a realistic description of the $\mathrm{cmc}$ and the aggregate size. The limits of the Yukawa model have been identified which is important for the use of such simplified models for future simulation studies of selfassembly of ionic amphiphiles and the development of simple mean-field models for these systems.

We observed that DTAC micelles remain spherical over the entire salt concentration range, in line with experimental results. In contrast, SDS micelles grow and possess oblate rather than prolate ellipsoidal shapes at the $\mathrm{cmc}$. This supports the predictions of Tanford ${ }^{79}$ of almost 30 years ago. We showed, however, that prolate ellipsoidal shapes or the formation of cylindrical micelles are likely at very high surfactant and salt concentrations, also in agreement with experimental observations. A quantitative study of the salt dependence that influences sphere-to-rod transitions will be the subject of a future study in which we will also investigate the process of micellar fusion in more detail.

The present study of micellization of surfactants in the presence of salt demonstrates the capability of the model to study more complex systems. Possible future studies include mixed surfactants, cosurfactants, or higher valence salts. Further application of this implicit-solvent model in the study of lipid molecules would provide an important step toward large-scale molecular simulations of such systems. It remains an open task to study salt effects and to explore the limits of the Yukawa model for these molecules. More broadly, this study illustrates the importance of ion correlations and shortcomings of mean-field treatment of salt effects for simulation studies of self-assembly in the presence of Coulombic interactions.

Acknowledgment. This publication is based on work supported by the Princeton Center for Complex Materials (Grant
NSF DMR 0213706), the Department of Energy (Grant DEFG02-01ER15121), and by Award No. KUS-CI-018-02, made by King Abdullah University of Science and Technology (KAUST). A.J. gratefully acknowledges financial support from the Deutsche Forschungsgemeinschaft.

\section{References and Notes}

(1) Israelachvili, J. N. Intermolecular and Surface Forces; Academic Press: Orlando, FL, 1985.

(2) Hunter, R. J. Foundations of Colloid Science, Vol. 1; Clarendon Press: Oxford, U.K., 1987.

(3) Rosen, M. J. Surfactants and Interfacial Phenomena, 2nd ed.; Wiley: New York, 1989.

(4) Tanford, C. The Hydrophobic Effect: Formation of Micelles and Biological Membranes, 2nd ed.; Krieger: Malabar, FL, 1991.

(5) Myers, D. Surfactant Science and Technology, 2nd ed.; VCH: New York, 1992.

(6) Evans, D. F.; Wennerstrom, H. The Colloidal Domain: Where Physics, Chemistry, Biology, and Technology Meet, 2nd ed.; Wiley-VCH: New York, 1999.

(7) McGrath, K. M.; Dabbs, D. M.; Yao, N.; Aksay, I. A.; Gruner, S. M. Science 1997, 277, 552.

(8) Faul, C. F. J.; Antonietti, M. Adv. Mater. 2003, 15, 673.

(9) Miller, K. W. Int. Rev. Neurobiol. 1985, 27, 1.

(10) Klein, M. L.; Shinoda, W. Science 2008, 321, 798.

(11) Jönsson, B.; Edholm, O.; Teleman, O. J. Chem. Phys. 1986, 85, 2259. 819.

(13) Schweighofer, K. J.; Essmann, U.; Berkowitz, M. J. Phys. Chem. B 1997, 101, 3793 .

(14) Maillet, J. B.; Lachet, V.; Coveney, P. V. Phys. Chem. Chem. Phys. 1999, 1, 5277.

(15) Boek, E. S.; Jusufi, A.; Löwen, H.; Maitland, G. C. J. Phys.: Condens. Matter 2002, 14, 9413.

(16) Shelley, J.; Watanabe, K.; Klein, M. L. Int. J. Quantum Chem. 1990, 38,103 .

(17) MacKerell, A. D. J. Phys. Chem. 1995, 99, 1846

(18) Bruce, C. D.; Berkowitz, M. L.; Perera, L.; Forbes, M. D. E. J. Phys. Chem. B 2002, 106, 3788.

(19) Bruce, C. D.; Senapati, S.; Berkowitz, M. L.; Perera, L.; Forbes, M. D. E. J. Phys. Chem. B 2002, 106, 10902.

(20) Rakitin, A. R.; Pack, G. R. J. Phys. Chem. B 2004, 108, 2712.

(21) Yoshii, N.; Okazaki, S. Chem. Phys. Lett. 2006, 426, 66.

(22) Sammalkorpi, M.; Karttunen, M.; Haataja, M. J. Phys. Chem. B 2007, 111, 11722 .

(23) Jorge, M. Langmuir 2008, 24, 5714.

(24) Shang, B. Z.; Wang, Z. W.; Larson, R. G. J. Phys. Chem. B 2008, 112,2888 .

(25) Gambu, I.; Roux, B. J. Phys. Chem. B 1997, 101, 6066.

(26) Böckmann, R. A.; Hac, A.; Heimburg, T.; Grubmüller, H. Biophys. J. 2003, 85, 1647.

(27) Gurtovenko, A. A. J. Chem. Phys. 2005, 122, 244902.

(28) Sachs, J. N.; Crozier, P. S.; Woolf, T. B. J. Chem. Phys. 2004, $121,10847$.

(29) Vernier, P. T.; Ziegler, M. J.; Sun, Y.; Gundersen, M. A.; Tieleman, D. P. Phys. Biol. 2006, 3, 233.

(30) Lee, S.-J.; Song, Y.; Baker, N. A. Biophys. J. 2008, 94, 3565.

(31) Vemperala, S.; Domene, C.; Klein, M. L Biophys. J. 2008, 94, 4260.

(32) Alexandridis, P.; Holzwarth, J. F.; Hatton, T. A. Langmuir 1993, 9, 2045.

(33) Drouffe, J.-M.; Maggs, A. C.; Leibler, S. Science 1991, 254, 1353.

(34) Shinto, H.; Morisada, S.; Miyahara, M.; Higashitani, K. Langmuir 2004, 20, 2017.

(35) Morisada, S.; Shinto, H.; Higashitani, K. J. Phys. Chem. B 2005, $109,11762$.

(36) Lazaridis, T.; Mallik, B.; Chen, Y. J. Phys. Chem. B 2005, 109, 15098.

(37) Cooke, I. R.; Kremer, K.; Deserno, M. Phys. Rev. E 2005, 72, 011506.

(38) Brannigan, G.; Philips, P. F.; Brown, F. L. H. Phys. Rev. E 2005, 72, 011915.

(39) Cheong, D. W.; Panagiotopoulos, A. Z. Langmuir 2006, 22, 4076. (40) Morisada, S.; Shinto, H.; Higashitani, K. J. Chem. Theory Comput. 2007, 3, 1163.

(41) Allen, E. C.; Rutledge, G. C. J. Chem. Phys. 2008, 128, 154115. (42) Jusufi, A.; Hynninen, A.-P.; Panagiotopoulos, A. Z. J. Phys. Chem. B 2008, 112, 13783.

(43) Walther, D.; Kuzmin, P.; Donath, E. Eur. Biophys. J. 1996, 24, 125 . 
(44) Bhattacharya, A.; Mahanti, S. D.; Chakrabarti, A. J. Chem. Phys. 1998, 108, 10281.

(45) Maiti, P. K.; Chowdhury, D. J. Chem. Phys. 1998, 109, 5126.

(46) Bhattacharya, A.; Mahanti, S. D. J. Phys.: Condens. Matter 2001 13,1413

(47) Tzlil, S.; Ben-Shaul, A. Biophys. J. 2005, 89, 2972.

(48) Mbamala, E. C.; Fahr, A.; May, S. Langmuir 2006, 22, 5129.

(49) Tzlil, S.; Murray, D.; Ben-Shaul, A. Biophys. J. 2008, 95, 1745

(50) Beard, D. A.; Schlick, T. Structure 2001, 9, 105.

(51) Khandogin, J.; Brooks, C. L., III. Biochemistry 2006, 45, 9363.

(52) Kim, Y. C.; Hummer, G. J. Mol. Biol. 2008, 375, 1416.

(53) Ferrenberg, A. M.; Swendsen, R. H. Phys. Rev. Lett. 1988, 61, 2635 .

(54) Panagiotopoulos, A. Z. J. Phys.: Condens. Matter 2000, 12, R25.

(55) Wilding, N. B. Am. J. Phys. 2001, 69, 1147.

(56) Floriano, M. A.; Caponetti, E.; Panagiotopoulos, A. Z. Langmuir 1999, 15, 3143.

(57) Siepmann, J. I.; Frenkel, D. Mol. Phys. 1992, 75, 59.

(58) Frenkel, D.; Smit, B. Understanding Molecular Simulation; Academic Press: New York, 1996.

(59) Stilbs, P.; Walderhaug, H.; Lindman, B. J. Phys. Chem. 1983, 87, 4762.

(60) Chandrasekhar, D.; Spellmeyer, D.; Jorgensen, W. L. J. Am. Chem. Soc. 1984, 106, 903.

(61) Lenart, P. J.; Jusufi, A.; Panagiotopoulos, A. Z. J. Chem. Phys. 2007, 126, 044509.

(62) Weeks, J. D.; Chandler, D.; Andersen, H. C. J. Chem. Phys. 1971, $54,5237$.

(63) Chatterjee, A.; Moulik, S. P.; Sanyal, S. K.; Mishra, B. K.; Puri, P. M. J. Phys. Chem. B 2001, 105, 12823.
(64) Ropers, M. H.; Czichocki, G.; Brezesinski, G. J. Phys. Chem. B 2003, 107,5281

(65) Hoyer, H. W.; Marmo, A. J. Phys. Chem. 1961, 65, 1807.

(66) Mehta, S. K.; Bhasin, K. K.; Kumar, A.; Dham, S. Colloids Surf., A 2006, 278, 17.

(67) Thévenot, C.; Grassl, B.; Bastiat, G.; Binana, W. Colloids Surf., A 2005, 252, 105 .

(68) Umlong, I. M.; Ismail, K. Colloids Surf., A 2007, 299, 8.

(69) Roelants, E.; De Schryver, F. C. Langmuir 1987, 3, 209.

(70) Emerson, M. F.; Holtzer, A. J. Phys. Chem. 1967, 71, 1898.

(71) Berr, S. S.; Jones, R. R M.; Johnson, J. S., Jr. J. Phys. Chem. 1992, 96,5611

(72) Barry, B. W.; Russel, G. F. J. J. Colloid Interface Sci. 1972, 40, 174.

(73) Hoyer, H. W. J. Phys. Chem. 1957, 61, 1283.

(74) Quina, F. H.; Nassar, P. M.; Bonilha, P. M.; Bales, B. L. J. Phys. Chem. 1995, 99, 17028.

(75) Bales, B. L.; Zana, R. J. Phys. Chem. B 2002, 106, 1926.

(76) Drummond, C. J.; Grieser, F.; Healy, T. W. Chem. Phys. Lett. 1987, $140,493$.

(77) Shanks, P. C.; Franses, E. I. J. Phys. Chem. 1992, 96, 1794

(78) Vass, S.; Pedersen, J. S.; Plestil, J.; Laggner, P.; Rétfalvi, E.; Varga, I.; Gilányi, T. Langmuir 2008, 24, 408.

(79) Tanford, C. J. Phys. Chem. 1980, 76, 1972.

(80) Griffiths, P. C.; Paul, A.; Heenan, R. K.; Penfold, J.; Ranganathan, R.; Bales, B. L. J. Phys. Chem. B 2004, 108, 3810.

(81) Berr, S. S.; Jones, R. R. M. Langmuir 1988, 4, 1247.

(82) Ozeki, S.; Ikeda, S. Bull. Chem. Soc. Jpn. 1981, 54, 552.

JP901032G 